\title{
Alprazolam Measurement
}

National Cancer Institute

\section{Source}

National Cancer Institute. Alprazolam Measurement. NCI Thesaurus. Code C75370.

The determination of the amount of alprazolam present in a sample. 